\title{
Nesting material enrichment reduces severity of overgrooming-related self-injury in individually housed rats
}

\author{
Shaun Yon-Seng Khoo $\circledast^{1,2}$, Vanessa Correia ${ }^{1}$ and Alexandra Uhrig ${ }^{1}$
}

${ }^{1}$ Center for Studies in Behavioral Neurobiology, Department of Psychology, Concordia University, Montreal, Quebec, Canada. and ${ }^{2}$ Present Address: Department of Pharmacology and Physiology, Faculty of Medicine, Université de Montréal, Montreal, QC, Canada.

*shaun.khoo@umontreal.ca

\begin{abstract}
Individual or singly-housing laboratory rats is common in many animal facilities, but has an adverse impact on the welfare of this social species. It has previously been shown that a small proportion of individually-housed mice ( $5 \%$ ) engage in pathological overgrooming behaviour, but this has not been assessed in rats. We performed an observational study to determine the prevalence of overgrooming-related self-injury and whether providing nesting material enrichment throughout an animal's life would affect the prevalence or severity of overgrooming-related self-injury. Due to protocol differences between projects in our behavioural neuroscience lab, unenriched rats received a nylabone and a shelter $(n=167)$, while baseline-enriched rats received a nylabone, shelter, and shredded paper nesting material throughout experiments $(n=238)$. Unenriched rats received nesting material enrichment after the onset of overgrooming-related self-injury. Over 18 months, rats were monitored by their experimenters on a daily basis (5-7 days/week over 2-3 months/project) and any cases of overgrooming-related self-injury were recorded. Replicating the findings of previous studies in mice, we observed 20 cases of overgrooming-related self-injury ( $5 \%$ ) with no difference in prevalence between rats on the basis of supplier, cage position, experimental procedure (behavioural only or involving surgerical procedures), reinforcer (ethanol or sugar), or level of baseline-enrichment. While there was no difference in onset severity between rats that were unenriched at baseline and baseline-enriched rats, baseline-enriched rats had lower self-injury severity scores at 1, 2, and 4-week follow-ups. These results suggest that nesting material enrichment provided throughout an animal's life may reduce overgrooming-related self-injury.
\end{abstract}

Key words: Grooming; Nesting; Social Behaviour; Housing; Refinement

\section{Introduction}

Laboratory rats have been domesticated for nearly two centuries, but they have retained a highly social nature.[1, 2] Despite the importance of social interaction for laboratory rats $[1,3]$, it is often necessary to individually house rats for experimental purposes, such as to observe certain behavioural patterns or to prevent damage to implants. For example, individual housing is necessary to measure the alcohol intake of individual animals in their home-cage[4] and is used in animal models of alcohol addiction because it can promote alcohol consumption. $[5,6]$ In the neuroscience literature, rats are frequently described as individually housed. For example, we briefly surveyed the Journal of Neuroscience and found individual housing reported in approximately half of the papers published in 2017 that describe using rats and their housing conditions (Supplementary Figure S1).[7]

However, individual housing has adverse psychological effects on rats, promoting depression-like symptoms and neurological changes.[8] Moreover, stress and anxiety can contribute towards aberrant self-grooming behaviour in rodents[9] and we have observed on several occasions that an animal's overgrooming has resulted in alopecia or skin lesions. We attribute these occurrences to individual housing because they are not observed in group-housed rats in our laboratory or in group-housed rats in our animal facility. Repetitive aberrant behaviours, or stereotypies, are thought of to be an indicator of poor welfare.[10] Previous studies that observed individually and group-housed rats have found that individually housed rats are less active but engage in more self-directed behaviours, 
Table 1. Number of rats monitored (and overgrooming-related self-injury cases) according to supplier, reinforcer, experimental procedures, and baseline enrichment

\begin{tabular}{|c|c|c|c|c|}
\hline Supplier* & Reinforcer & $\begin{array}{c}\text { Experimental } \\
\text { Procedures }\end{array}$ & Baseline Enrichment & Number of Rats (Cases) \\
\hline Charles River & Sugar & Behaviour & Yes & $118(6)$ \\
\hline Charles River & Sugar & Behaviour & No & 30 (None) \\
\hline Charles River & Sugar & Surgery & Yes & $44(4)$ \\
\hline Charles River & Sugar & Surgery & No & $59(1)$ \\
\hline Charles River & Ethanol & Behaviour & No & $24(4)$ \\
\hline Harlan Laboratories & Ethanol & Behaviour & No & $48(3)$ \\
\hline Breeding Colony & Ethanol & Behaviour & Yes & $10(1)$ \\
\hline Breeding Colony & Ethanol & Surgery & Yes & $76(1)$ \\
\hline Breeding Colony & Ethanol & Surgery & No & 6 (None) \\
\hline
\end{tabular}

* Information on whether rats were littermates was not obtained from commercial suppliers. Breeding colony rats were maintained on a background of Long Evans rats from both Charles River and Harlan Laboratories.

such as pawing and tail manipulation.[11, 12] This is consistent with studies in mice that have found that the prevalence of selfbarbering was $5.7 \%$ in individually housed mice and $<1 \%$ in group-housed mice.[13]

Self-injury and aberrant or pathological grooming behaviour is used as an animal model of various psychological disorders, such as anxiety[14], obsessive-compulsive disorder[13, 15, 16], autism spectrum disorders[17], Tourette syndrome[18], and trichotillomania[13, 19]. Active self-injury behaviours such as overgrooming can be induced by administration of psychostimulants, such as caffeine or pemoline, and it is thought that these experiments model aspects of autism spectrum disorders or anxiety where self-injury or repetitive behaviours are prevalent.[14, 20, 21] Loss of histamine signaling in the tuberomammillary nucleus of the hypothalamus, a dysregulation similar to Tourette syndrome, results in pathological grooming in mice.[18] Similarly, the Shank3 mutant mouse which is bred to model a genetic autism spectrum disorder (22q13 deletion syndrome), spontaneously displays pathological grooming behaviour.[17] The Long-Evans rats used in our laboratory are an outbred strain, so it seems most likely that social isolation stress rather than a genetic or physiological pathology is the cause of the overgrooming-related self-injury we have observed.

Environmental enrichment has many beneficial effects for laboratory animals. In disease models, environmental enrichment can delay or prevent symptoms of Huntington's disease[22-24], Alzheimer's disease[25, 26], schizophrenia[27], stress-induced anxiety[28], or depression.[29] In individually housed rats, environmental enrichment in the form of providing structures, chew toys, and nesting material has been shown to reduce stress as measured by adrenal size or the levels of adrenocorticotropic hormone and corticosterone.[30, 31] Nesting material may be especially beneficial because rats show a preference for environments with shredded paper or nesting boxes, but not for other standard enrichment items like PVC tunnels or wooden sticks.[32]

We therefore conducted an observational study that aimed to assess the prevalence of overgrooming-related self-injury and examine whether environmental enrichment with nesting material could reduce the severity or prevalence of overgrooming-related self-injury. While all rats that presented symptoms of overgrooming-related self-injury were provided with additional nesting material enrichment once experimenters identified the onset of overgrooming-related selfinjury, differences in experimental protocols between projects resulted in some rats receiving nesting material as part of their standard housing throughout their presence in our facility (baseline-enriched rats), while other rats did not (unen- riched rats). We hypothesised that rats provided with baseline enrichment would have lower prevalence and reduced severity of overgrooming-related self-injury. We also tested several alternative explanations including cage location (top, middle or bottom)[33], experimental procedures (behavioural experiments or experiments involving surgical procedures), reinforcer type (ethanol or sugar) and animal supplier (Charles River, Harlan Laboratories or an internal breeding colony).

\section{Methods}

\section{Animals}

Animals across multiple projects involving individual housing of rats were monitored over 18 months. Rats were monitored for the entirety of their projects, each of which was 2-3 months in duration. All rats $(\mathrm{N}=415)$ were housed in standard plastic cages $(44.5 \times 25.8 \times 21.7 \mathrm{~cm})$ containing Teklad Sani Chip bedding (Cat\#: 7090, Envigo, QC, Canada). The vivarium was climate-controlled $\left(21^{\circ} \mathrm{C} \pm 2^{\circ} \mathrm{C}\right)$ on a $12 \mathrm{~h}: 12 \mathrm{~h}$ light/dark cycle (lights on at 07:00). Male Long-Evans rats were purchased from Charles River $(n=275)$ or Harlan Laboratories (Envigo; $\mathrm{n}=48$ ) weighing 220-240g on arrival, or bred in-house ( $\mathrm{n}$ =92) for use in experiments that were approved by the Animal Research Ethics Committee at Concordia University and performed in accordance with guidelines from the Canadian Council on Animal Care. Hygiene monitoring was performed by Animal Care Facility staff.

In these experiments, rats were individually housed for the purpose of measuring home-cage consumption of ethanol ( $\mathrm{n}$ = 164) or sugar solutions $(n=251)$. Rats from Harlan Laboratories were used for ethanol experiments because previous studies have shown that they drink more ethanol in Pavlovian conditioning experiments (see Table 1 for distribution of rats across study conditions).[34] Rats were subjected to either behavioural experiments involving appetitive conditioning $(n=230$, 'behavioural rats') or experiments that involved high-impact surgical procedures to perform viral microinjections or implant guide cannulae or optic fibres prior to behavioural procedures ( $\mathrm{n}=185$, 'surgery rats').[35-37] Rats that underwent surgery received isoflurane anaesthesia and analgesia with ketoprofen and buprenorphine.[35-37] Project and protocol differences meant that some rats were provided with a minimal level of baseline enrichment (unenriched rats, Figure 1A), which included a nylabone (Cat\#: K3580, Bio-Serv, NJ, USA) and, in most cases, a shelter (Cat\#: K3245 or K3325 or $\mathrm{K} 3365$, Bio-Serv). For example, unenriched rats were primarily rats in ethanol-based experiments due to concerns that enrichment may reduce ethanol consumption (Table 1).[38, 39] 

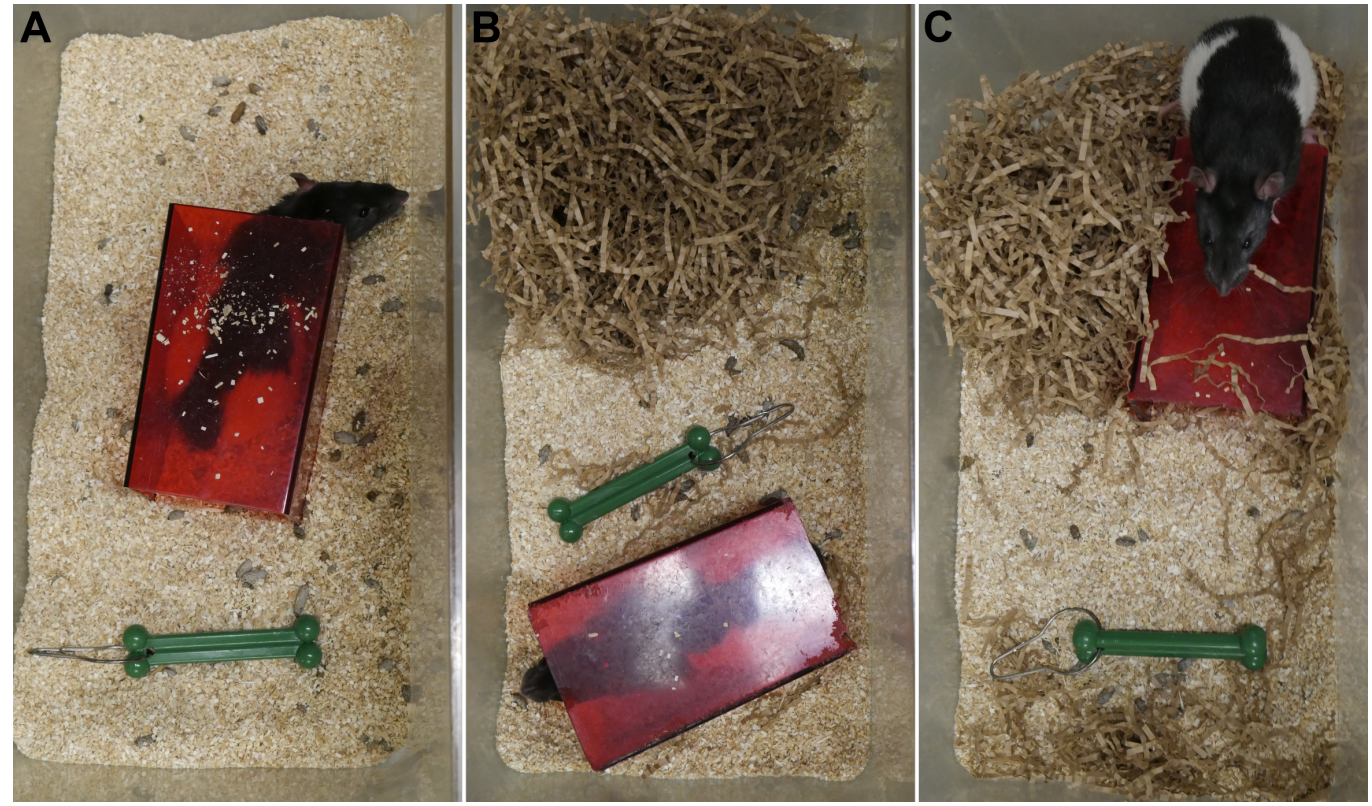

Figure 1. Nesting material allowed rats to build more complex environments in their home-cage. (a) Unenriched rats were maintained in standard housing included a nylabone chew toy, which was normally attached to the cage lid, and a shelter. (b) Baseline-enriched rats were also provided with shredded paper, which they used to build nests. (c) In some cases, rats used both the shelter and nesting material together. After the onset of symptoms, unenriched rats were provided with the same level of enrichment as baseline-enriched rats.

Baseline-enriched rats were provided additional nesting enrichment throughout experiments, including a nylabone, a shelter, and shredded paper (EnviroDri ${ }^{\circledR}$, FiberCore, OH, USA \& Shepherd Specialty Papers, TN, USA) that enabled them to nest and build a more complex environment (Figure 1B-C). Experimenters $(n=7)$ were trained to monitor rats for signs of overgrooming-related self-injury and, where self-injury was observed, to score the degree of injury on a 7-point scale (see Table 2 and Figure 2). Experimenters were advised that the aim of the study was to identify any protocol differences that may affect overgrooming-related self-injury. Most experimenters were involved in monitoring rats with and without enrichment and observations were usually made in pairs due to shared responsibilities for each project. In all experiments, animals were handled 5-7 days a week for behavioural experiments, during which time they were weighed and monitored daily. Self-injury severity scores were recorded at the onset of symptoms and then at 1, 2, and 4 weeks after onset. Unenriched rats were provided with nesting material enrichment after the onset of symptoms. Due to the study's observational nature, all comparisons between baseline-enriched and unenriched rats are between groups.

\section{Data Analysis and Availability}

Data were analysed using SPSS 24 (IBM, NY, USA). To determine if prevalence was associated with particular factors, $\chi^{2}$ tests were performed. Severity scores from onset to the 2-week follow-up were analysed using mixed-design ANOVA with week as a within-subjects factor and baseline-enrichment, cage location, experimental procedures, reinforcer (ethanol or sugar), or supplier as a between-subjects factor. Because 3 rats had completed behavioural experiments before week 4, this week was analysed separately using an independent samples t-test or one-way ANOVA. All post-hoc tests were Bonferronicorrected. Because the factors of experimental procedures, reinforcer type, and supplier frequently biased whether rats received baseline-enrichment or not (Table 1), we used a mixed linear model to statistically control for bias. We used a scaled identity covariance matrix type and maximum likelihood estimation to model whether the fixed effects of baselineenrichment, experimental procedures, reinforcer, and supplier affected severity scores from onset to week 2 when all cases were available for analysis.[40] Data are presented as means \pm SEM. Raw data underlying is available on Figshare.[7]

\section{Results}

\section{Prevalence of Overgrooming-related Self-injury}

We observed a total of 20 cases of overgrooming-related selfinjury ( $5 \%$ of monitored rats). Mean \pm SEM (SD) weight at on-

Table 2. Scoring criteria for overgrooming-related self-injury

\begin{tabular}{|c|c|}
\hline Severity Score & Criteria \\
\hline o - Not present & $\begin{array}{l}\text { No sign of overgrooming and skin has } \\
\text { recovered. Hair regrowth is present }\end{array}$ \\
\hline 1 - Very mild & $\begin{array}{l}\text { Hair loss to an area representing }<1 \\
\mathrm{~cm}^{2} \text {, skin intact }\end{array}$ \\
\hline 2 - Mild & $\begin{array}{l}\text { Hair loss to an area representing }>1 \\
\mathrm{~cm}^{2} \text {, skin intact }\end{array}$ \\
\hline 3 - Moderate & $\begin{array}{l}\text { Hair loss }>1 \mathrm{~cm}^{2} \text { with skin } \\
\text { inflammation or lesions (e.g. red or } \\
\text { raised skin) but no bleeding }\end{array}$ \\
\hline 4 - Somewhat severe & $\begin{array}{l}\text { Skin lesion }>1 \mathrm{~cm}^{2} \text { with little to no } \\
\text { bleeding }\end{array}$ \\
\hline 5 - Severe & $\begin{array}{l}\text { Skin lesion with broken skin and } \\
\text { bleeding }\end{array}$ \\
\hline 6 - Very severe & $\begin{array}{l}\text { Warrants immediate euthanasia. } \\
\text { Animals with open wounds were taken } \\
\text { to Animal Care Facility/veterinary } \\
\text { staff for assessment to determine an } \\
\text { appropriate course of action. This } \\
\text { never resulted in a euthanasia } \\
\text { recommendation during the course of } \\
\text { the study. }\end{array}$ \\
\hline
\end{tabular}



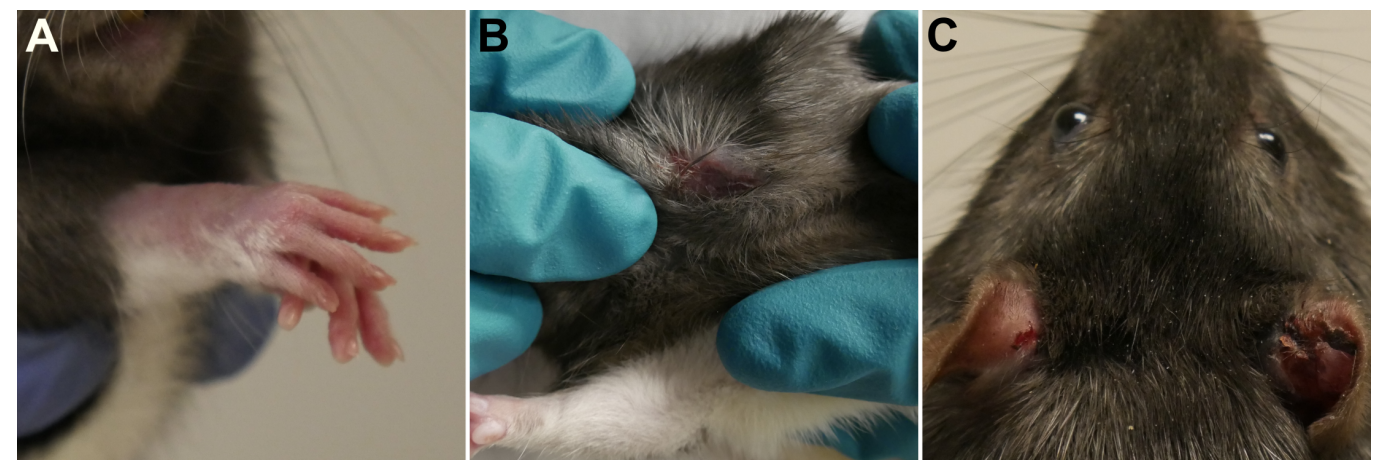

Figure 2. Typical presentation of overgrooming-related self-injury was focused on the anterior portion of the body. (a) A mild case of forepaw overgrooming involving hair loss only (severity score = 2). (b) A moderate case of overgrooming on the animal's neck with some skin lesioning $($ severity score $=3$ ). (c) A severe case affecting both ears (severity score $=5$ ).

set of symptoms was $367 \pm 10.4(46.7) \mathrm{g}$, ranging from $264 \mathrm{~g}$ to $476 \mathrm{~g}$. Overgrooming-related self-injury typically presented as alopecia (in mild cases) or as skin lesions (in moderate-severe cases) on the neck or shoulders ( $45 \%$ of cases), forepaws ( $25 \%$ ), around the ears $(25 \%)$, and more rarely on the snout $(10 \%)$ or tail $(5 \%)$. Percentages sum to more than $100 \%$ because two cases involved multiple affected areas (1 case affecting the paws and shoulders, 1 case affecting the paws and tail).

The prevalence of overgrooming was not associated with any specific factor. Prevalence did not appear to be associated with whether rats received a high baseline level of enrichment $(\mathrm{n}=248$ rats $)$ or were unenriched $(\mathrm{n}=167)$ at baseline $(\chi 2(1)=$ $0.001, p=0.982$; Figure $3 \mathrm{~A}$ ). Previous studies suggest that cage location may affect animal welfare because cages positioned higher on the racks may receive more light that stresses the animals,[33] so we also examined cage location. In our vivarium, each wall is fitted with 4 racks and rats are positioned so that they are distributed equally across racks. We found a distribution of overgrooming cases among the top $(n=4)$, middle two racks $(n=11)$, and bottom racks $(n=5)$ that was not associated with cage location $\times 2(2)=0.344, p=0.842$; Figure $3 B$ ). Rats were subjected to either behavioural experiments with no surgery ( $n=230,14$ cases of overgrooming) or to experiments involving surgical procedures such as viral microinjections, implantation of chronically indwelling cannulae, or optic fibres ( $\mathrm{n}$ $=185,6$ cases of overgrooming), but experimental procedure was not associated with an increased likelihood of engaging in overgrooming $\left(\chi_{2}(1)=1.808, p=0.179\right.$; Figure $\left.3 \mathrm{C}\right)$. Rats that were used in sugar studies $(\mathrm{n}=251,11$ cases) were not more likely to present with overgrooming-related self-injury than rats in ethanol studies $(n=162,9$ cases; $\chi 2(1)=0.264, p=$ 0.607; Figure 3D). All three sources of rats were also affected (Figure 3E), with 15 cases in Charles River rats, 3 cases in Harlan rats and 2 cases in rats bred in-house. There was no association between the supplier of the animals and the prevalence of overgrooming $(x 2(2)=1.86, \mathrm{p}=0.395)$.

\section{Severity of Overgrooming-related Self-injury}

Rats that received baseline nesting material enrichment as part of their standard housing $(n=12)$ experienced less severe self-injury and a more rapid recovery than unenriched rats $(\mathrm{n}=8$; Figure $4 \mathrm{~A})$ that were only provided nesting material enrichment after the onset of overgrooming-related selfinjury. A mixed-design ANOVA with baseline-enrichment as a between-subjects factor and week as a within-subjects factor found that baseline-enriched rats had significantly lower selfinjury severity scores $(F(1,18)=15.243, p=0.001)$. Although there was no main effect of week $(\mathrm{F}(1.481,26.666)=2.694, \mathrm{p}$ $=0.099$, Greenhouse-Geisser $\epsilon=0.741)$, there was a signifi- cant week $\times$ baseline-enrichment interaction $(\mathrm{F}(1.481,26.666)$ $=5.468, \mathrm{p}=0.017)$. Bonferroni-corrected post-hoc comparisons revealed that while there was no significant difference between baseline-enriched and unenriched rats at onset ( $p$ $=0.179)$, baseline-enriched rats had significantly lower selfinjury severity scores at the 1 -week follow-up $(\mathrm{p}<0.001)$ and the 2-week follow-up ( $p=0.002)$, perhaps because the unenriched rats increased in severity. At the 4-week follow-up, 11 baseline-enriched rats and 6 unenriched rats were still undergoing experiments. An independent samples t-test showed that the difference in severity was still statistically significant $(\mathrm{t}(15)=2.278, \mathrm{p}=0.038)$.

An alternative hypothesis might be that cage position influences the severity of overgrooming-related self-injury, but this was not the case. A mixed-design ANOVA with cage position as a between-subjects factor and week as a withinsubjects factor found no main effect of cage position $(\mathrm{F}(2,17)$ $=0.456, p=0.641 ;$ Figure $4 \mathrm{~B})$. Severity scores did not significantly change over the two weeks of follow-ups $(\mathrm{F}(2,34)$ $=1.896, \mathrm{p}=0.166)$ and there was no significant week $\times$ position interaction $(\mathrm{F}(4,34)=1.205, \mathrm{p}=0.327)$. At week 4 , when there were 17 rats remaining in experiments (top $n=3$, middle $n=10$, bottom $n=4$ ), a one-way ANOVA found no significant effect of position $(\mathrm{F}(2,14)=0.663, \mathrm{p}=0.531)$.

We also found no difference between behavioural experiments and experiments involving surgery (Figure 4C). A mixeddesign ANOVA with experimental procedure as a betweensubjects factor and week as a within-subjects factor found no main effect of experimental procedure $(\mathrm{F}(1,18)=2.992, \mathrm{p}$ $=0.101)$, week $(\mathrm{F}(1.468,26.418)=1.876, \mathrm{p}=0.18, \epsilon=0.734)$ or week $\times$ experimental procedure interaction $(\mathrm{F}(1.468,26.418)$ $=1.5, \mathrm{p}=0.24)$. One reviewer has suggested that the nonsignificant main effect of experimental procedure $(p=0.101)$ may be indicative of a trend or weak effect. We therefore examined the Bonferroni-corrected post-hoc comparisons at each timepoint, which indicated no significant difference between behavioural and surgery rats at onset $(p=0.481)$, week $1(p=$ $0.056)$ or week $2(p=0.13)$. At the 4 -week follow-up, there were 11 behavioural rats and 6 surgery rats remaining and an independent $\mathrm{t}$-test showed no significant difference between their severity scores $(t(15)=0.873, p=0.396)$.

Rats in studies with a sugar reinforcer presented with significantly lower severity scores than rats in ethanol studies (Figure $4 \mathrm{D} ; \mathrm{F}(1,18)=11.722, \mathrm{p}=0.003)$. However, mixed-design ANOVA found no significant effect of week $(\mathrm{F}(1.52,27.377)=$ 2.179, $\mathrm{p}=0.142, \epsilon=0.76)$ or week $\times$ reinforcer interaction $(\mathrm{F}(1.52,27.377)=2.326, \mathrm{p}=0.128)$. At week 4 , there were 7 rats in ethanol studies and 10 rats in sugar studies and rats in the sugar studies had significantly lower severity scores $(\mathrm{t}(15)$ $=2.601, \mathrm{p}=0.02$ ). 


\section{A \\ Enrichment}

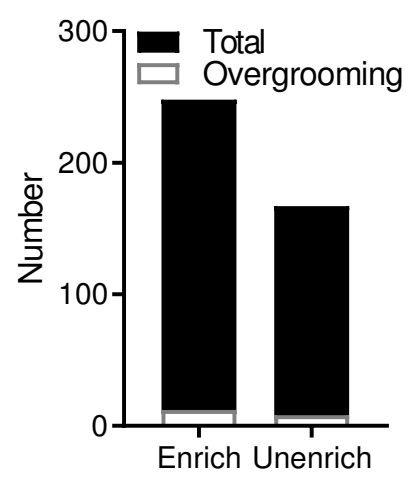

Proportion of Total

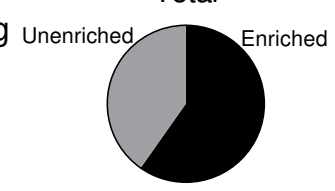

Proportion Overgrooming Unenriched Enriched

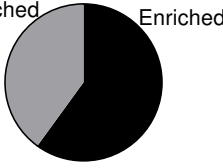

C Experimental Procedures
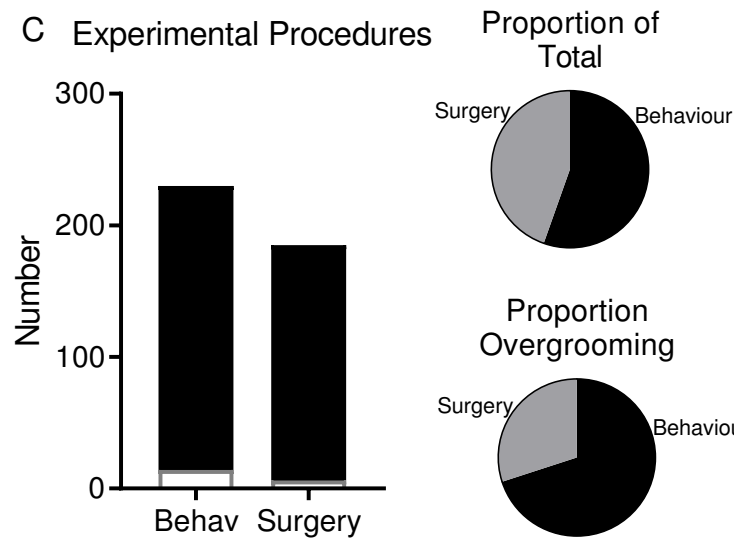

\section{Proportion} Overgrooming

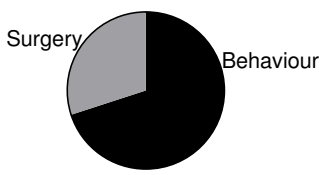

E

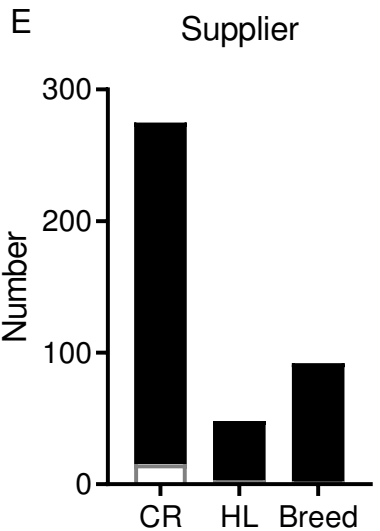

\section{B Cage Location}

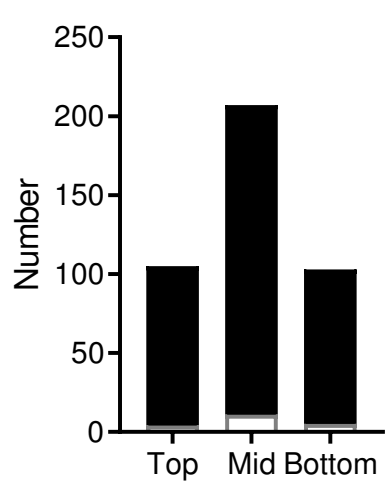

Proportion of Total

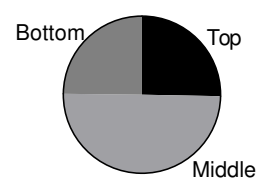

Proportion

Overgrooming
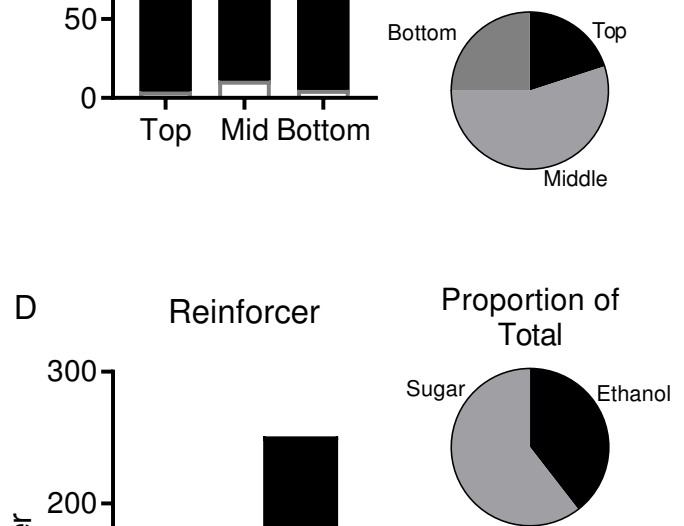

Proportion Overgrooming

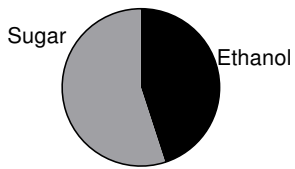

Ethanol Sugar

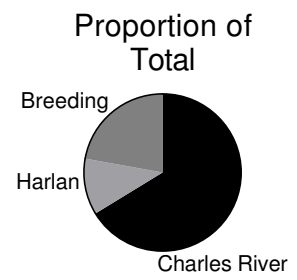

Proportion

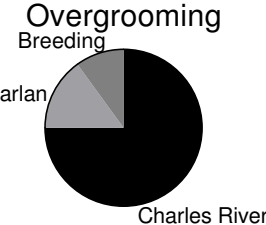

Figure 3. Prevalence was not associated with any specific factor. (a) Baseline-enriched rats that received nesting material enrichment throughout experiments had a similar prevalence to unenriched rats who received nesting material enrichment only after the onset of symptoms. (b) Cage location, (c) experimental procedures (behavioural experiments only or experiments including intracranial surgery), (d) reinforcer type (ethanol or sugar), and (e) supplier were all unassociated with prevalence. Data are presented as frequencies with proportions of the total and proportion presenting with overgrooming-related self-injury. CR: Charles River; HL: Harlan Laboratories

Supplier did not have a clear effect on severity (Figure 4E). Mixed-design ANOVA found no main effect of supplier $(\mathrm{F}(2,17)$ $=0.601, \mathrm{p}=0.559)$, week $(\mathrm{F}(1.499,25.475)=1.755, \mathrm{p}=0.197$, $\epsilon=0.749)$, or week $\times$ supplier interaction $(F(2.997,25.475)=$ $0.845, \mathrm{p}=0.482$ ). However, prior to the 4 -week follow-up 3 Charles River rats completed their experiments, such that there were 12 Charles River rats, 3 Harlan, and 2 breeding colony rats remaining. A one-way ANOVA found a significant effect of supplier $(\mathrm{F}(2,14)=4.941, \mathrm{p}=0.024)$ and Bonferronicorrected post-hoc comparisons found Harlan rats had significantly higher severity scores than Charles River rats $(p=0.024)$, but there were no other significant pairwise comparisons $(p \geq$ 0.138 ). However, this result is confounded by the small number of rats obtained from Harlan laboratories and the breeding colony and because all of the Harlan rats were in the unenriched group. 
A

Enrichment

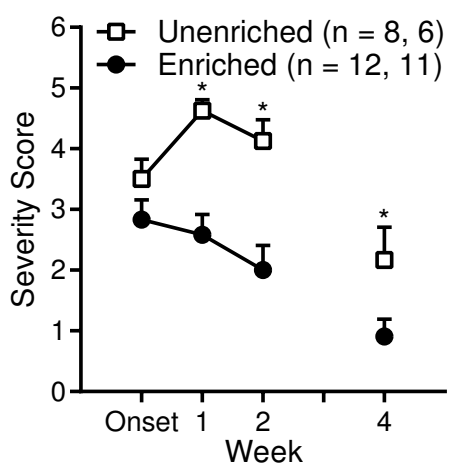

D

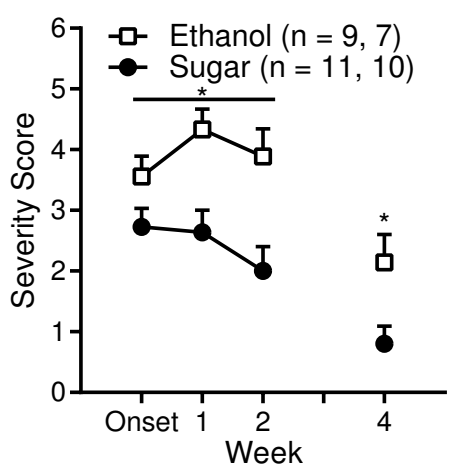

B

Cage Location

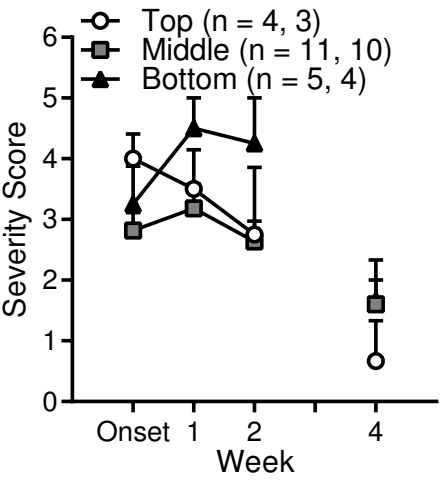

$\mathrm{E}$

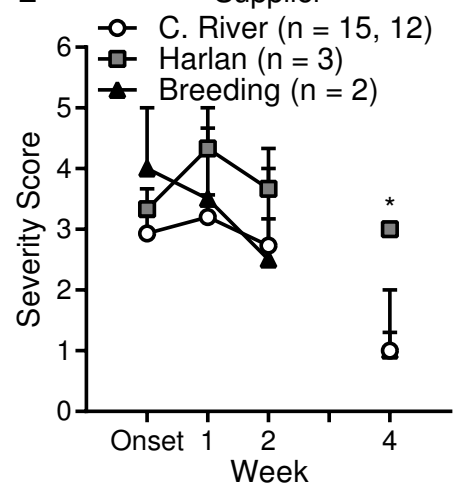

C Experimental Procedures

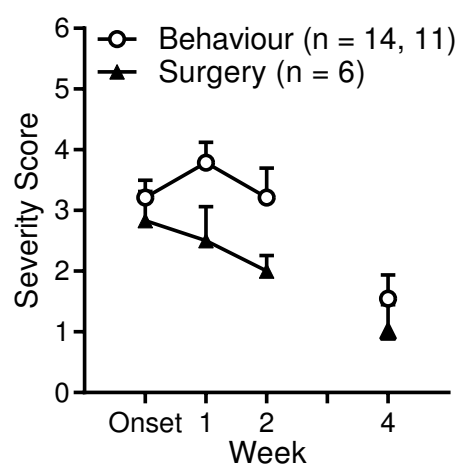

F Behaviour Rats Only

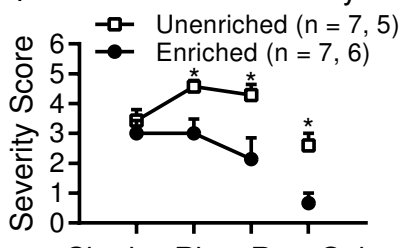

G Charles River Rats Only

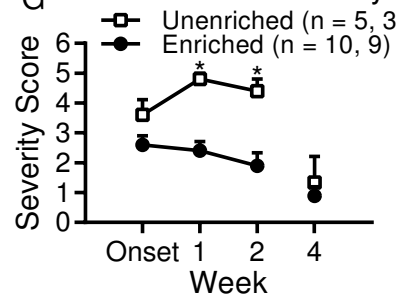

Figure 4. Provision of nesting material enrichment at baseline was associated with lower severity and improved recovery from overgrooming-related self-injury. (a) Rats that received baseline enrichment had similar self-injury severity scores at onset to unenriched rats that received nesting material enrichment only after onset. Baseline-enriched rats had significantly lower severity at one-, two- and four-week follow-ups. (b) The position of the cage did not affect the severity score at onset or at any point during follow-up. (c) There was also no difference between behavioural experiments and experiments involving surgery. (d) Prior to statistical control for confounds, rats with ethanol as a reinforcer had higher severity scores than sugar rats throughout the monitoring period. (e) There was no difference between suppliers at onset or one- or two-week follow-ups. At week 4, rats from Harlan had significantly higher severity scores than rats from Charles River or from the internal breeding colony. (f) The effect of baseline enrichment was robust when analysing rats from behavioural experiments alone or (g) Charles River rats alone. Data are mean \pm SEM and ns are given in legends for onset to week 2 and then for week 4 (if changed). *p<0.05 for Bonferroni-corrected post-hocs following mixed-design analysis of variance (ANOVA) at weeks 1-2. At week 4, data were analysed using an independent samples t-test or one-way ANOVA with Bonferroni-corrected post-hocs because not all rats were still undergoing experiments at this point.

\section{Statistical control of confounds}

Procedural differences related to experimental procedures (behavioural vs surgery), reinforcer type (ethanol vs sugar), and supplier frequently biased whether rats were in the baselineenriched group or unenriched group (Table 1). While experimental control for confounding variables is not possible in an observational study, we used a mixed linear model to test the main effects that predicted severity scores from onset to the 2-week follow-up, when all cases were available for analysis. A mixed linear model using a scaled identity covariance matrix, maximum likelihood estimation, and random intercept suggested that only baseline-enrichment was a significant predictor, with unenriched rats having higher severity scores than rats in the baseline-enriched group $(\beta=2.09 \pm 0.83, \mathrm{t}(20)=$ $2.52, \mathrm{p}=0.02$ ). Experimental procedures was included in the model because of the potential marginal difference at week 1 , but was not a significant predictor $(\beta=0.64 \pm 0.44, \mathrm{t}(20)$ $=1.46, \mathrm{p}=0.158$ ). Reinforcer type was included because it had significant effects when analysed alone, but was also not a significant predictor $(\beta=-0.31 \pm 0.94, \mathrm{t}(20)=-0.33, \mathrm{p}=$ 0.746). Supplier was also included because Harlan rats had significantly higher severity scores at week 4, which also appeared visually (but not statistically) in weeks 1-2. However, supplier was not a significant predictor because neither being from Charles River $(\beta=-1.41 \pm 1.13, \mathrm{t}(20)=-1.25, \mathrm{p}=0.226)$ or Harlan $(\beta=-1.96 \pm 1.15, \mathrm{t}(20)=-1.71, \mathrm{p}=0.102)$ predicted severity scores.

To further control for possible confounds of experimental procedure and supplier, we were also able to analyse behavioural rats (i.e. rats whose experimental procedures did not include surgeries) and Charles River rats separately. Both of these conditions had a sufficient number of cases that were distributed across baseline-enriched and unenriched conditions. This analysis was not possible for reinforcer type because only 1 sugar rat was unenriched and only 2 ethanol rats were baselineenriched.

Analysing behavioural rats alone (baseline-enriched $n=7$, unenriched $\mathrm{n}=7$; Figure $4 \mathrm{~F}$ ) with a mixed-design ANOVA, demonstrated that baseline-enrichment was significantly associated with lower severity scores $(\mathrm{F}(1,12)=6.536, \mathrm{p}=0.025)$. While there was no significant effect of week $(F(2,24)=2.446$, $\mathrm{p}=0.108)$, there was a significant week $\times$ baseline-enrichment interaction $(\mathrm{F}(2,24)=4.28, \mathrm{p}=0.026)$, with Bonferroni post- 
hocs showing that baseline-enriched rats had lower severity scores at the 1-week $(p=0.012)$ and 2 -week $(p=0.019)$ followups. An independent t-test also showed a significant difference at week $4(t(9)=3.747, p=0.005$; baseline-enriched $n=$ 6 , unenriched $\mathrm{n}=5$ ).

Similarly, analysing Charles River rats alone (baselineenriched $n=10$, unenriched $=5$, Figure $4 \mathrm{G}$ ), showed there was statistically significant effect of baseline-enrichment on severity scores $(F(1,13)=19.414, p=0.001)$. There was a significant week $\times$ baseline-enrichment interaction $(F(2,26)=3.586$, $\mathrm{p}=0.042)$, but no main effect of week $(\mathrm{F}(2,26)=1.546, \mathrm{p}=$ 0.232). Bonferroni-corrected post-hoc comparisons showed baseline-enriched rats had lower severity scores at the 1-week $(\mathrm{p}<0.001)$ and 2 -week $(\mathrm{p}=0.003)$ follow-ups. At the $4-$ week follow-up there were only 3 unenriched and 9 baselineenriched Charles River rats with no significant difference in their severity scores $(t(10)=0.62, p=0.549)$.

\section{Discussion}

The present study found that rats that received nesting material enrichment at baseline experienced less severe overgrooming-related self-injury than rats that did not receive baseline-enrichment. Although there was no difference in prevalence between rats that received nesting material enrichment at baseline compared to rats that were unenriched at baseline, the difference in severity and more rapid recovery after onset suggests that the simple refinement of providing shredded paper as nesting material provides a measurable improvement in the welfare of individually housed rats.

Numerous studies have shown that environmental enrichment is beneficial for laboratory rats. Our results are consistent with previous reports that environmental enrichment can reduce stress, anxiety, and depression-like symptoms in laboratory animals, though we do not claim to have directly measured these behaviours.[2, 23-28, 30, 31, 41] They further demonstrate that even a relatively small improvement in the level of enrichment can reduce the severity of overgroomingrelated self-injury and thus likely increases welfare.[10] Nesting material enrichment may be a particularly important form of environmental enrichment because instead of being a relatively static structure, it can serve multiple needs. Previous studies have shown that mice given shredded paper nesting material are able to build nests that are comparable to their wild counterparts.[42] While a shelter can provide a good hiding place (note that in Figure 1, all of the rats are in or on their tunnels), in group-housed mice it can actually promote aggression.[43] In addition to a hiding place, nesting material can provide thermal insulation[42] and allows animals to express more of their natural behaviours, an important 'five freedoms' consideration.[44] In mice, nesting material reduced aggression between group-housed animals.[43] In rats, using nesting material is also a learned behaviour, which may explain why providing additional enrichment at the onset of overgrooming-related self-injury did not produce an immediate reduction in severity.[45] There is also the possibility that rats that were unenriched at baseline and given nesting material after onset experienced some neophobia towards the novel material, although several studies suggest that laboratory rats show low levels of neophobia that last no more than a day.[1, 46, 47] Moreover, when rats are introduced to a clean cage with new bedding, physiological effects only last a few hours while rats in our study were monitored over weeks.[4850] It seems more likely that unenriched rats developed habitual stereotypies that persist even after their home-cage conditions were improved.[10] Shredded paper nesting material may therefore be a particularly effective environmental enrichment for reducing stress in laboratory rats and should be provided wherever possible throughout the animal's life.

Importantly, these effects appeared to be independent of several other factors, such as whether experimental procedures were purely behavioural or also included intracranial surgery. There was no significant effect of experimental procedure on the prevalence or severity of overgrooming-related self-injury. At most, there may have been a difference at the 1-week followup, but this is probably due to chance because mixed linear modelling did not indicate any effect of experimental procedure. Moreover, if this was a real effect it would require a much larger sample size (at least 34 rats for $80 \%$ power according to $G^{*}$ Power 3.1.9.4)[51] to detect. It would also be highly counterintuitive because, despite the putative impact of intracranial surgeries, it would suggest that intracranial surgeries might reduce the severity of overgrooming-related self-injury. One potential explanation for the lack of a difference between behavioural and surgery rats is that surgery rats are provided a higher level of care, handling, and habituation to experimental procedures that compensate for the stress associated with intracranial surgery experiments. Regardless of whether surgical experimental procedures affect the severity of overgroomingrelated self-injury, our data suggests that baseline nesting material enrichment reduces the overgrooming-related selfinjury because even when behavioural rats are analysed separately, the effect of baseline-enrichment is still statistically significant.

There also did not appear to be a specific age at which rats would present with overgrooming-related self-injury because the weight at onset varied widely between $264 \mathrm{~g}$, corresponding to a time shortly after arrival, and $476 \mathrm{~g}$ corresponding to several weeks after arrival. Cage location also did not appear to have an effect since there was no significant different in prevalence or severity at any point in time.

Individual housing of laboratory rats remains an important welfare concern for scientists and animal welfare specialists. Baseline environmental enrichment had no effect on the prevalence or onset severity of overgrooming-related selfinjury. This suggests that individual housing remains a key stressor for laboratory rats and it is notable that the observed $5 \%$ prevalence in our study is almost identical to the previously observed $5.7 \%$ prevalence of self-barbering in individually housed mice.[13] It also appears that enrichment needs to be permanent in order for it to have beneficial effects on the severity of overgrooming-related self-injury over time. In our study, rats that were observed to overgroom were provided with additional enrichment, but they still did not recover at the same rate as rats that had nesting material enrichment at baseline. Indeed, it appears that their condition initially worsened and at 4 weeks, while nearly half of the baseline-enriched rats had fully recovered $(n=5)$, only 1 of the rats that was unenriched at baseline had fully recovered. This suggests that environmental enrichment works by modulating the stress response over a long period of time.

One limitation of the present study is that we are unable to determine the nature of any proximal cause that might trigger overgrooming-related self-injury. We have observed, consistent with previous reports[13], a relatively low prevalence of overgrooming-related self-injury. There appears to be no consistent age/weight, experimental procedure, cage location, or supplier associated with the onset of overgrooming. The low prevalence of overgrooming limits the forms of study primarily to observational designs because it requires a large number of animals to accumulate sufficient cases. This also results in a potential confound because it may be the case that projects where experimenters provided nesting material enrichment at baseline were less stressful than experiments without enrichment at baseline. Experiments using a sugar rein- 
forcer tended to involve baseline enrichment while ethanol experiments did not. It is theoretically possible that the mere exposure to ethanol increased the severity of overgroomingrelated self-injury despite ethanol's anxiolytic effects, but this does not seem likely because all ethanol consumption was voluntary, and during Pavlovian conditioning, only small amounts of ethanol are available that are unlikely to have a long-lasting effect.[37, 52] While mixed linear modelling suggested that baseline enrichment was the more important factor, this type of confound invites replication of the present study in other laboratories.

Another possibility is that our findings were affected by genetic differences between animals. For example, the significant difference between Harlan rats and Charles river rats at the 4-week follow-up is confounded because all 3 Harlan rats were also unenriched rats, so it is unclear whether they had higher severity scores because they were Harlan rats or because they were unenriched until the onset of overgrooming-related self-injury. Although previous studies have found differences between Charles River rats and Harlan rats[34, 53], it has not been shown whether this extends to pathological grooming behaviour. Mixed linear modelling did not identify supplier or reinforcer as a significant predictors of severity scores. Moreover, even if Charles River rats are analysed alone, there is still a statistically significant effect of baseline enrichment, suggesting that the effect of baseline enrichment is unlikely to be a simple vendor difference.

Inter-experimenter differences are also a possible confound because it was not possible to calculate inter-rater reliability after training, due to the infrequent nature of cases. However, observations were frequently confirmed by a second experimenter, because most projects involved multiple experimenters. Ideally, the causes and best practices for management of overgrooming could be examined by direct experimental manipulation of self-injury behaviour. While this could be achieved using pharmacological approaches, such as the pemoline model[14, 20, 21], it is unclear whether this would accurately model the overgrooming that results from individual housing. Therefore, while it is difficult to draw conclusions that would be as strong as those drawn from an experimental study, observational studies of overgrooming-related selfinjury and its management are currently the most practical approach.

\section{Conclusions}

Individual housing of laboratory rats presents an important welfare concern for scientists. While individual housing may be justifiable in certain experimental models, it appears to result in approximately $5 \%$ of animals engaging in pathological overgrooming resulting in self-injury. Prevalence and severity appear to be independent of several other factors, such as the location of the cage, experimental procedures, and source of the animals. While reinforcer type biased whether rats were in the baseline-enrichment group or unenriched until onset of symptoms, statistical analysis suggested that baseline enrichment was the more important factor. Thus, the simple refinement of providing rats with shredded paper nesting material enrichment throughout their life in the animal facility, in addition to shelters and chew toys, can reduce the severity of self-injury and promote recovery.

\section{Acknowledgements}

The authors gratefully acknowledge all experimenters in the Chaudhri laboratory who carefully monitored their animals during the study period: Milan Valyear, Franz Villaruel, Mandy Rita Lecocq, Alexa Brown and Ghislaine Deyab.

\section{Conflict of Interest Declaration}

The authors have no conflicts of interest to declare.

\section{Funding}

This research was supported by grants from the Canadian Institutes of Health Research (CIHR; MOP-137030; primary investigator: Nadia Chaudhri) and the Natural Sciences and Engineering Research Council (NSERC; RGPIN-2017-04802; N.C.). S.Y.K. was supported by a Concordia Horizon Postdoctoral Fellowship and a postdoctoral fellowship from Fonds de Recherche du Québec - Santé. A.U. received an undergraduate summer research award from the Department of Psychology at Concordia University.

\section{References}

1. Barnett SA. The rat: A study in behavior. Revised ed. Canberra: Australian National University Press; 1976.

2. Makowska IJ, Weary DM. The importance of burrowing, climbing and standing upright for laboratory rats. Royal Society Open Science. 2016;3(6):160136. doi: 10.1098/rsos.160136.

3. Burn CC. What is it like to be a rat? Rat sensory perception and its implications for experimental design and rat welfare. Applied Animal Behaviour Science. 2008;112(1-2):132. doi: 10.1016/j.applanim.2008.02.007.

4. Wise RA. Voluntary ethanol intake in rats following exposure to ethanol on various schedules. Psychopharmacologia. 1973;29(3):203-210. doi: 10.1007/bfo0414034.

5. Deatherage G. Effects of housing density on alcohol intake in the rat. Physiology \& Behavior. 1972;9(1):55-57. doi: 10.1016/0031-9384(72)90264-8.

6. McCool BA, Chappell AM. Early social isolation in male long-evans rats alters both appetitive and consummatory behaviors expressed during operant ethanol selfadministration. Alcoholism: Clinical and Experimental Research. 2009;33(2):273-282. doi: 10.1111/j.15300277.2008.00830.x.

7. Khoo SYS, Correia V, Uhrig A. Nesting material enrichment reduces severity of overgrooming-related selfinjury in individually housed rats. Figshare. 2019;doi: 10.6084/m9.figshare.7716578.

8. Gilles YD, Polston EK. Effects of social deprivation on social and depressive-like behaviors and the numbers of oxytocin expressing neurons in rats. Behavioural Brain Research. 2017;328:28-38. doi: 10.1016/j.bbr.2017.03.036.

9. Kalueff AV, Stewart AM, Song C, Berridge KC, Graybiel AM, Fentress JC. Neurobiology of rodent self-grooming and its value for translational neuroscience. Nature Reviews Neuroscience. 2015;17:45-59. doi: 10.1038/nrn.2015.8.

10. Mason GJ, Latham NR. Can't stop, won't stop: Is stereotypy a reliable animal welfare indicator? Animal Welfare. 2004;13(1):57-69.

11. Baenninger LP. Comparison of behavioural development in socially isolated and grouped rats. Animal Behaviour. 1967;15(2):312-323. doi: 10.1016/0003-3472(67)90018-8.

12. Hurst JL, Barnard CJ, Nevison CM, West CD. Housing and welfare in laboratory rats: Welfare implications of isolation and social contact among caged males. Animal Welfare. 1997;6(4):329-347. 
13. Garner JP, Weisker SM, Dufour B, Mench JA. Barbering (fur and whisker trimming) by laboratory mice as a model of human trichotillomania and obsessive-compulsive spectrum disorders. Comparative Medicine. 2004;54(2):216224.

14. Yuan X, Devine DP. The role of anxiety in vulnerability for self-injurious behaviour: studies in a rodent model. Behavioural Brain Research. 2016;311:201-209. doi: 10.1016/j.bbr.2016.05.041.

15. Welch JM, Lu J, Rodriguiz RM, Trotta NC, Peca J, Ding JD, et al. Cortico-striatal synaptic defects and OCD-like behaviours in Sapap3-mutant mice. Nature. 2007;448(7156):894-900. doi: 10.1038/nature06104.

16. Ahmari SE, Dougherty DD. Dissecting OCD circuits: From animal models to targeted treatments. Depression and Anxiety. 2015;32(8):550-562. doi: 10.1002/da.22367.

17. Peça J, Feliciano C, Ting JT, Wang W, Wells MF, Venkatraman TN, et al. Shank3 mutant mice display autistic-like behaviours and striatal dysfunction. Nature. 2011;472(7344):437-442. doi: 10.1038/nature09965.

18. Rapanelli M, Frick L, Bito H, Pittenger C. Histamine modulation of the basal ganglia circuitry in the development of pathological grooming. Proceedings of the National Academy of Sciences. 2017;114(25):6599-6604. doi: 10.1073/pnas.1704547114.

19. Chen SK, Tvrdik P, Peden E, Cho S, Wu S, Spangrude $\mathrm{G}$, et al. Hematopoietic origin of pathological grooming in Hoxb8 mutant mice. Cell. 2010;141(5):775-785. doi: 10.1016/j.cell.2010.03.055.

20. Mueller K, Hollingsworth E, Pettit H. Repeated pemoline produces self-injurious behavior in adult and weanling rats. Pharmacology Biochemistry and Behavior. 1986;25(5):933-938. doi: 10.1016/0091-3057(86)90065-1.

21. Kies SD, Devine DP. Self-injurious behaviour: a comparison of caffeine and pemoline models in rats. Pharmacology Biochemistry and Behavior. 2004;79(4):587-598. doi: 10.1016/j.pbb.2004.09.010.

22. van Dellen A, Blakemore C, Deacon R, York D, Hannan AJ. Delaying the onset of Huntington's in mice. Nature. 2000;404(6779):721-722. doi: 10.1038/35008142.

23. Mo C, Hannan AJ, Renoir T. Environmental factors as modulators of neurodegeneration: Insights from geneenvironment interactions in Huntington's disease. Neuroscience \& Biobehavioral Reviews. 2015;52:178-192. doi: 10.1016/j.neubiorev.2015.03.003.

24. Mo C, Renoir T, Hannan AJ. What's wrong with my mouse cage? Methodological considerations for modeling lifestyle factors and gene-environment interactions in mice. Journal of Neuroscience Methods. 2016;265:99-108. doi: 10.1016/j.jneumeth.2015.08.008.

25. Fischer A. Environmental enrichment as a method to improve cognitive function. What can we learn from animal models? NeuroImage. 2016;131:42-47. doi: 10.1016/j.neuroimage.2015.11.039.

26. Herring A, Ambrée O, Tomm M, Habermann H, Sachser $\mathrm{N}$, Paulus W, et al. Environmental enrichment enhances cellular plasticity in transgenic mice with Alzheimer-like pathology. Experimental Neurology. 2009;216(1):184-192. doi: 10.1016/j.expneurol.2008.11.027.

27. Bator E, Latusz J, Wędzony K, Maćkowiak M. Adolescent environmental enrichment prevents the emergence of schizophrenia-like abnormalities in a neurodevelopmental model of schizophrenia. European Neuropsychopharmacology. 2018;28(1):97-108. doi: 10.1016/j.euroneuro.2017.11.013.

28. Novaes LS, dos Santos NB, Batalhote RFP, Malta MB, Camarini R, Scavone C, et al. Environmental enrichment protects against stress-induced anxiety: Role of glucocorti- coid receptor, ERK, and CREB signaling in the basolateral amygdala. Neuropharmacology. 2017;113:457-466. doi: 10.1016/j.neuropharm.2016.10.026.

29. Pang TY, Du X, Catchlove WA, Renoir T, Lawrence AJ, Hannan AJ. Positive environmental modification of depressive phenotype and abnormal hypothalamic-pituitary-adrenal axis activity in female $\mathrm{C} 57 \mathrm{BL} / 6 \mathrm{~J}$ mice during abstinence from chronic ethanol consumption. Frontiers in Pharmacology. 2013;4:93. doi: 10.3389/fphar.2013.00093.

30. Abou-Ismail UA, Mahboub HD. The effects of enriching laboratory cages using various physical structures on multiple measures of welfare in singly-housed rats. Laboratory Animals. 2011;45(3):145-153. doi: 10.1258/la.2011.010149.

31. Belz EE, Kennell JS, Czambel RK, Rubin RT, Rhodes ME. Environmental enrichment lowers stress-responsive hormones in singly housed male and female rats. Pharmacology Biochemistry and Behavior. 2003;76(3):481-486. doi: 10.1016/j.pbb.2003.09.005.

32. Patterson-Kane EG, Harper DN, Hunt M. The cage preferences of laboratory rats. Laboratory Animals. 2001;35(1):74-79. doi: 10.1258/0023677011911390.

33. Castelhano-Carlos MJ, Baumans V. The impact of light, noise, cage cleaning and in-house transport on welfare and stress of laboratory rats. Laboratory Animals. 2009;43(4):311-327. doi: 10.1258/la.2009.0080098.

34. Sparks LM, Sciascia JM, Ayorech Z, Chaudhri N. Vendor differences in alcohol consumption and the contribution of dopamine receptors to pavlovian-conditioned alcohol-seeking in Long-Evans rats. Psychopharmacology. 2014;231(4):753-764. doi: 10.1007/s00213-013-3292-5.

35. Khoo SYS, LeCocq MR, Deyab GE, Chaudhri N. Context and topography determine the role of basolateral amygdala metabotropic glutamate receptor 5 in appetitive Pavlovian responding. Neuropsychopharmacology. 2019;44:15241533. doi: 10.1038/s41386-019-0335-6.

36. Villaruel FR, Lacroix F, Sanio C, Sparks DW, Chapman CA, Chaudhri N. Optogenetic activation of the infralimbic cortex suppresses the return of appetitive pavlovianconditioned responding following extinction. Cerebral Cortex. 2018;28(12):4210-4221. doi: 10.1093/cercor/bhx275.

37. LeCocq MR, Lahlou S, Chahine M, Padillo LN, Chaudhri N. Modeling relapse to pavlovian alcohol-seeking in rats using reinstatement and spontaneous recovery paradigms. Alcoholism: Clinical and Experimental Research. 2018;42(9):1795-1806. doi: 10.1111/acer.13825.

38. Deehan Jr GA, Plamatier MI, Cain ME, Kiefer SW. Differential rearing conditions and alcohol-preferring rats: Consumption of and operant responding for ethanol. Behavioral Neuroscience. 2011;125(2):184-193. doi: 10.1037/a0022627.

39. Ehringer MA, Hoft NR, Zunhammer M. Reduced alcohol consumption in mice with access to a running wheel. Alcohol. 2009;43(6):443-452. doi: 10.1016/j.alcohol.2009.06.003.

40. Heck RH, Thomas SL, Tabata LN. Multilevel and longitudinal modeling with IBM SPSS. 2nd ed. New York: Routledge; 2014. doi: 10.4324/9780203701249.

41. Makowska IJ, Weary DM. Differences in anticipatory behaviour between rats (rattus norvegicus) housed in standard versus semi-naturalistic laboratory environments. PLoS ONE. 2016;11(1):e0147595. doi: 10.1371/journal.pone.0147595.

42. Hess SE, Rohr S, Dufour BD, Gaskill BN, Pajor EA, Garner JP. Home improvement: $\mathrm{C} 57 \mathrm{BL} / 6 \mathrm{~J}$ mice given more naturalistic nesting materials build better nests. Journal of the American Association for Laboratory Animal Science : JAALAS. 2008;47(6):25-31. 
43. Van Loo PLP, Kruitwagen CLJJ, Koolhaas JM, Van de Weerd HA, Van Zutphen LFM, Baumans V. Influence of cage enrichment on aggressive behaviour and physiological parameters in male mice. Applied Animal Behaviour Science. 2002;76(1):65-81. doi: 10.1016/S0168-1591(01)00200-3.

44. Brønstad A, Newcomer CE, Decelle T, Everitt JI, Guillen J, Laber K. Current concepts of harm-benefit analysis of animal experiments - report from the AALASFELASA working group on harm-benefit analysis - part 1. Laboratory Animals. 2016;50(1_suppl):1-20. doi: $10.1177 / 0023677216642398$.

45. Van Loo PLP, Baumans V. The importance of learning young: the use of nesting material in laboratory rats. Laboratory Animals. 2004;38(1):17-24. doi: 10.1258/00236770460734353.

46. Barnett SA. Experiments on 'neophobia' in wild and laboratory rats. British Journal of Psychology. 1958;49(3):195201. doi: 10.1111/j.2044-8295.1958.tbo0657.x.

47. Stryjek R, Modlińska K, Pisula W. Species specific behavioural patterns (digging and swimming) and reaction to novel objects in wild type, wistar, sprague-dawley and brown norway rats. PLoS ONE. 2012;7(7):e40642. doi: 10.1371/journal.pone.0040642.

48. Burn CC, Peters A, Mason GJ. Acute effects of cage cleaning at different frequencies on laboratory rat behaviour and welfare. Animal Welfare. 2006;15(2):161-171.

49. Burn CC, Mason GJ. Effects of cage-cleaning frequency on laboratory rat reproduction, cannibalism, and welfare. Applied Animal Behaviour Science. 2008;114(1-2):235-247. doi: 10.1016/j.applanim.2008.02.005.

50. Burn CC, Mason GJ. Rats seem indifferent between their own scent-marked homecages and clean cages. Applied Animal Behaviour Science. 2008;115(3-4):201-210. doi: 10.1016/j.applanim.2008.06.002.

51. Faul F, Erdfelder E, Lang AG, Buchner A. G*Power 3: a flexible statistical power analysis program for the social, behavioral, and biomedical sciences. Behavior Research Methods. 2007;39(2):175-91. doi: 10.3758/BF03193146.

52. Khoo SY, Sciascia JM, Pettorelli A, Maddux JMN, Chaudhri $\mathrm{N}$. The medial prefrontal cortex is required for responding to alcohol-predictive cues but only in the absence of alcohol delivery. Journal of Psychopharmacology. 2019;33:842854. doi: 10.1177/0269881119844180.

53. Fitzpatrick CJ, Gopalakrishnan S, Cogan ES, Yager LM, Meyer PJ, Lovic V, et al. Variation in the form of pavlovian conditioned approach behavior among outbred male Sprague-Dawley rats from different vendors and colonies: Sign-tracking vs. goal-tracking. PLoS ONE. 2013;8(10):e75042. doi: 10.1371/journal.pone.0075042. 
Journal of Neuroscience Papers that Report Individually Housing Rats

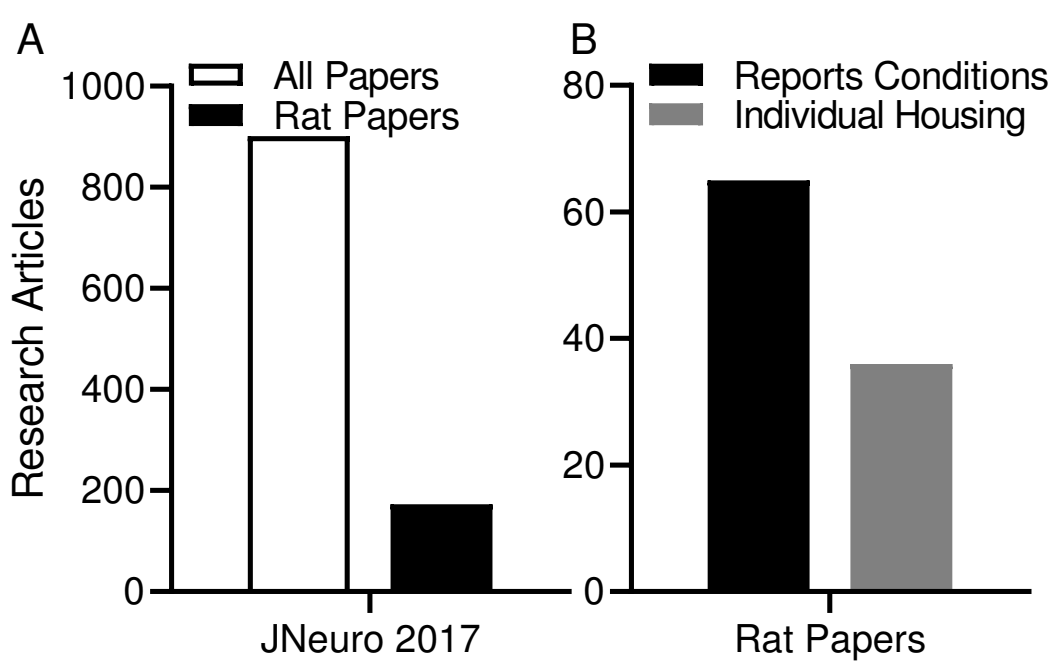

Figure S1. Individual housing is prevalent in neuroscience research. (A) In 2017, approximately one-fifth of research articles in the Journal of Neuroscience reported using rats as a model animal. (B) Of the 65 rat papers that reported housing conditions, $55 \%$ reported using individual housing during part or all of theirexperiments. Data from Khoo, Correia, and Uhrig (2019), Figshare. doi:10.6084/m9.figshare.7716578. 